\title{
Silicoaluminophosphate (SAPO)-Templated Activated Carbons
}

\author{
Yunxiang Li, ${ }^{\dagger}$ Xia Wang, ${ }^{\dagger}$ Thomas Thersleff, Gunnar Svensson, and Niklas Hedin*(i) \\ Department of Materials and Environmental Chemistry, Stockholm University, SE-10691 Stockholm, Sweden
}

\section{Supporting Information}

ABSTRACT: Microporous activated carbon was prepared by depositing and pyrolyzing propylene within the microporous voids of SAPO-37 and subsequently removing the template by a treatment with $\mathrm{HCl}$ and $\mathrm{NaOH}$. The carbon had a high surface area and large micropore and ultramicropore volumes. The yield, crystallinity, morphology, and adsorption properties compared well with those of a structurally related zeolite-Ytemplated carbon. No HF was needed to remove the SAPO37 template in contrast to the zeolite $\mathrm{Y}$ template, which could be of industrial importance.

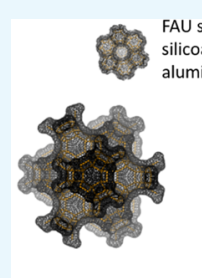

SAPO-37/Activated carbon $(A C)$ composite
FAU structure, e.g.,

silicoaluminophosphate (SAPO)-37, uminosilicate zeolite $Y$,

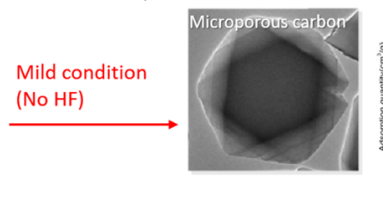

SAPO-37 templated AC

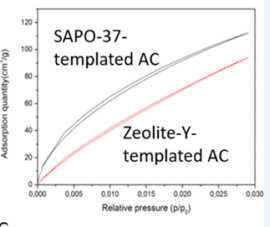

Higher $\mathrm{CO}_{2}$ uptake

\section{INTRODUCTION}

Activated carbons (ACs) are commercialized adsorbents for water and gas purification and electrode materials in doublelayer-based supercapacitors, and the corresponding research fields are active. ${ }^{1-3}$ There has been a growing interest in preparing three-dimensionally connected ACs with regularly arrayed micropores using zeolites as templates. ${ }^{4,5}$ Because of the interconnectivities, such ACs are expected to have high electric conductivities and are attractive for use in applications that demand high conductivities and capacitances. ${ }^{5-8}$

Zeolite-templated carbons are, in general, prepared by first depositing and pyrolyzing carbon precursors within the zeolite and then dissolving the zeolite template. ${ }^{9-11}$ Zeolites are crystalline and microporous (pores $<2 \mathrm{~nm}$ ) aluminosilicates with general structures described by three-letter framework-type codes. $^{12,13}$ Furfuryl alcohol, propylene, acetylene, and acetonitrile are examples of suitable carbon precursors, and FAU, MOR, LTA, BEA, and MFI types of zeolites have been used as templates. $^{14-18}$

A stoichiometric removal of the zeolite framework is normally promoted by $\mathrm{HF}$ (or sometimes by $\mathrm{NaOH}$ ) under hydrothermal conditions; ${ }^{19-21}$ however, as HF imposes safety hazards, it limits the options for industrialization. With hydrothermal treatment using $\mathrm{NaOH}$, zeolites are sometimes dissolved and recrystallized. For instance, Tendeloo et al. showed that a commercial $\mathrm{NH}_{4}{ }^{+}-\mathrm{Y}$ zeolite transformed into five different framework types depending on the cations of the base; however, with $\mathrm{NaOH}$, the zeolite $\mathrm{Y}$ framework largely persisted. ${ }^{22}$

The large class of crystalline and microporous aluminophosphates $\left(\mathrm{AlPO}_{4} \mathrm{~s}\right)^{23}$ is isoelectronic to microporous silicates $\left(\mathrm{SiO}_{2}\right)$, with alternating and oxygen-linked aluminum and phosphorous atoms. Their frameworks are mainly covalently bonded and have no net charge. However, the chemistry of $\mathrm{AlPO}_{4} \mathrm{~s}$ and microporous silicates $\left(\mathrm{SiO}_{2}\right)$ is different and $\mathrm{AlPO}_{4} \mathrm{~s}$ can be dissolved in aqueous solutions of $\mathrm{HCl}^{24-26}$ Even though this chemical difference is promising for the use of $\mathrm{AlPO}_{4} \mathrm{~s}$ to derive ACs, only a limited amount of work has been performed.
One previous study has shown that carbon nanotubes can be synthesized within the channels of an $\mathrm{AlPO}_{4}-5$ material. ${ }^{27}$ However, its one-dimensional pore system makes it less useful for the synthesis of ACs, which requires at least two-dimensional pore systems with large pore window apertures and high thermal stabilities. ${ }^{16}$ These requirements for suitability met in a related class of templates, the microporous silicoaluminophosphates (SAPOs). Similar to zeolites (aluminosilicates), SAPOs have a negatively charged framework, but Si (formal charge of 4+) replaces $\mathrm{P}$ (formal charge of $5+$ ). ${ }^{28}$

FAU-type zeolites have been particularly successful in synthesizing zeolite-templated AC; ${ }^{15,17,29-34}$ however, microporous $\mathrm{AlPO}_{4} \mathrm{~s}$ with FAU frameworks have not been synthesized yet. SAPO-37 is FAU-structured. ${ }^{35,36}$ In addition to having a three-dimensional structure, large pores, and pore window apertures, $\mathrm{H}-\mathrm{SAPO}-37$ is highly acidic and its $-\mathrm{OH}$ groups are thermally stable. ${ }^{37} \mathrm{H}$-SAPO-37 is thermally stable up to $1000{ }^{\circ} \mathrm{C}$ but becomes amorphous in an ambient atmosphere when being exposed to water vapor. ${ }^{38}$ The high thermal stability and acidity of H-SAPO-37 are advantageous for infiltration and deposition of carbon precursors at high temperatures. Its low hydrothermal stability, on the other hand, makes it promising for dissolution under mild conditions. ${ }^{39,40} \mathrm{Wu}$ et al. ${ }^{41}$ used sulfuric acid as a catalyst, sucrose as a carbon precursor, and SAPO-11 and SAPO-34 as agents to achieve mesoporous carbons. To the best of our knowledge, we here present, for the first time, the synthesis of AC derived from the microporous interiors of a SAPO, whose framework could be removed under mild conditions without using HF.

\section{RESULTS AND DISCUSSION}

Figure 1 shows the powder X-ray diffraction (PXRD) patterns of the as-made SAPO-37 templates, the template-AC composites,

Received: January 15, 2019

Accepted: May 15, 2019

Published: June 7, 2019 


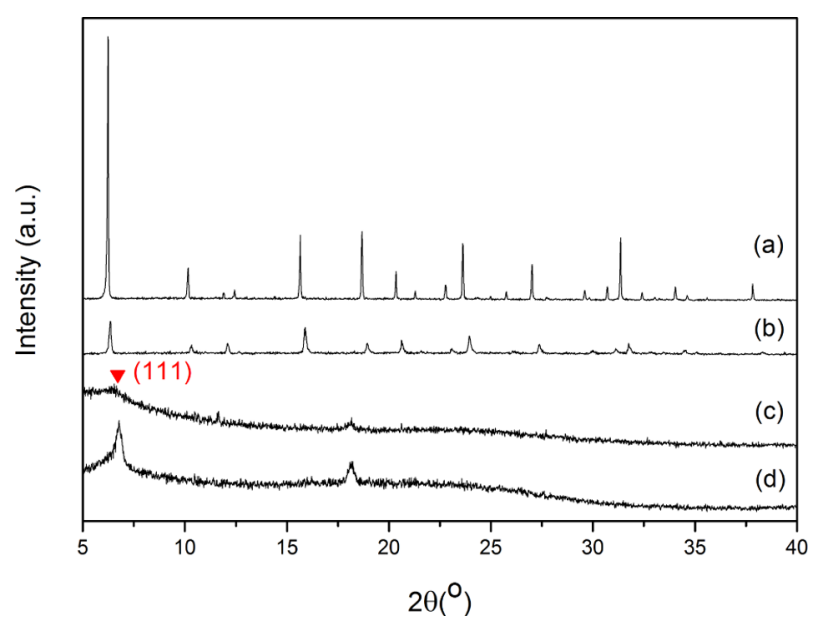

Figure 1. Powder X-ray diffraction patterns of the (a) as-made silicoaluminophosphate (SAPO)-37, (b) SAPO-37-activated carbon (AC) composite, (c) SAPO-37-templated AC, and (d) zeolite-Ytemplated AC.

and ACs. As can be seen in Figure 1a, the as-made SAPO-37 had a typical characteristic pattern of the FAU structure with narrow and intense peaks typical of a material of high crystallinity. The ACs were successfully prepared using the pure SAPO-37 and zeolite Y templates, as shown below.

The positions of the PXRD peaks shifted toward higher angles for the SAPO-37-AC composite (Figure 1b) as compared to those for the as-made SAPO-37 (Figure 1a), indicating that the SAPO-37 framework was well retained within the SAPO-37-AC composite after the propylene chemical vapor deposition (CVD) but the unit cell had shrunk slightly upon the thermal treatment. The SAPO-37-templated AC had one broad XRD diffraction peak (Figure 1c) at $2 \theta$ around $6^{\circ}$, which we attribute to the replication of the FAU framework 111. This peak is commonly visible also for zeolite-Y-templated ACs presented in the literature ${ }^{42,43}$ and is also detected in the reference material (zeolite-Y-templated AC), which had a comparably narrow peak at $2 \theta=6.8^{\circ}$ (Figure $1 \mathrm{~d}$ ). The reference material thus seems to display a higher degree of structural regularity than the SAPO37-templated AC. However, for both ACs, no peak at $2 \theta=\sim 22^{\circ}$ for layered graphite was observed in Figure 1c,d, suggesting that no or little carbon has been deposited on the external surfaces of the zeolite Y or SAPO-37 crystals during the propylene CVD. (Note that some allotments of the ACs had a diffraction peak at $2 \theta=18.2^{\circ}$, which was assigned to an impurity polytetrafluoroethylene. ${ }^{44}$ ) The particle morphologies of the templated ACs are relevant to understanding the mechanisms of formation and, potentially, their applications. Scanning electron microscopy (SEM) and transmission electron microscopy (TEM) were used to study the morphologies, and the results are shown in Figure 2. The crystals of SAPO-37 and the SAPO-37-templated AC (after the removal of the SAPO) have an octahedral shape (Figure $2 \mathrm{~b}, \mathrm{~d})$. The average particle size of $1180 \mathrm{~nm}$ for SAPO-37templated AC is smaller than that of $1690 \mathrm{~nm}$ for the SAPO-37 template, which is attributed to the SAPO-37 unit cell shrinkage during thermal treatment as discussed for the XRD peak shift. Moreover, the surfaces of the SAPO-37-templated AC appear smooth from the TEM image in Figure 2d (and its SEM image in Figure S1a as well), in contrast to the rough surfaces of carbon materials that have been ascribed to core-shell morphologies for $\mathrm{AlPO}_{4}$-5-templated carbons. ${ }^{45}$ This morphology-based information supports the hypothesis that the carbon has been

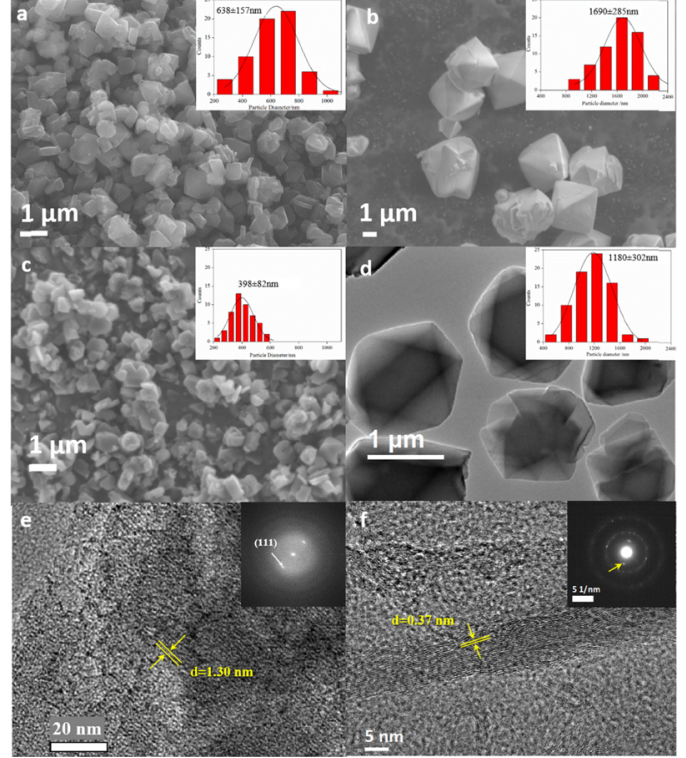

Figure 2. Scanning electron microscopy (SEM) images and particle size distributions of (a) as-made zeolite $\mathrm{Y}$, (b) silicoaluminophosphate (SAPO)-37, and (c) zeolite-Y-templated activated carbon (AC) and (d) transmission electron microscopy (TEM) image and particle size distribution of SAPO-37-templated AC. High-resolution TEM images of (e) zeolite-Y-templated AC and its Fourier transform (inset) and (f) SAPO-37-templated AC and its diffraction pattern (inset).

deposited within the nanometer-sized cages of the SAPO-37 crystals. The conserved octahedral morphology in the SAPO-37templated AC strongly supports the hypothesis that the propylene CVD functioned well and that the carbon precursor has penetrated well into the SAPO-37 template. Similar findings were observed for the zeolite $\mathrm{Y}$ and its corresponding zeolite-Ytemplated AC in Figure 2a,c. The average crystal size of zeolite $\mathrm{Y}$ was smaller than that of SAPO-37 (compare the features in Figure 2a,b). Likewise, the morphology of the zeolite Y crystals was also conserved in the zeolite-Y-templated AC.

High-resolution TEM is a well-established method to study features of zeolite-Y-templated $\mathrm{AC}^{42,46-50}$ and Figure $2 \mathrm{e}$ displays the HRTEM image of the zeolite-Y-templated AC. Although some defects were observed in the images of the carbon network, regular ordered lattice fringes were also observed, confirming that the zeolite-Y-templated AC retained at least some of the organized porous network of the zeolite $\mathrm{Y}$ template. The measured interfringe distance was $1.30 \pm 0.01$ $\mathrm{nm}$, in agreement with the $d$ value calculated from the powder diffraction peak ( $2 \theta$ at $6.8^{\circ}$ for $\mathrm{Cu} \mathrm{K} \alpha$ radiation). The Fourier transform of this image exhibits a pair of spots at the positions corresponding to the 111 reflection of zeolite $\mathrm{Y}$ (see the inset in Figure 2e). For the SAPO-37-templated AC, no obvious regular structure with respect to a replication of the FAU framework in the plane of (111) can be observed by TEM (Figure S1b). This is consistent with the broad powder diffraction peak $(2 \theta)$ at $6.8^{\circ}$ in Figure 1c. As shown in Figure 2f, ordered structures are observed in some areas of the HRTEM images of the SAPO-37templated AC; however, the relative amounts of these structures appear to be too low to display defined peaks in the PXRD pattern. For these features, the interspace distance calculated from the reflections as marked by the arrow in the SAED patterns is $0.37 \mathrm{~nm}$, which is close to the interlayer distance $d_{002}$ of turbostratic carbon and carbon black. ${ }^{51-56}$ Additionally, TEM-EDS analysis indicates that the ACs are relatively pure, as 
only small amounts of the SAPO-37 and zeolite Y matrices were detected in the corresponding ACs (see Table S1 and Figure S2 in the Supporting Information), although the TEM-EDS analysis included carbon signal from the holy carbon-coated grid unavoidably.

Electron energy-loss spectroscopy (EELS) can be used to determine the graphitization degree or the fraction of $\mathrm{sp}^{2} / \mathrm{sp}^{3}$ carbon in carbon materials. ${ }^{57-60}$ In this work, the fraction of $\mathrm{sp}^{2} /$ $\mathrm{sp}^{3}$ carbon for the ACs was estimated by EELS by analyzing the near-edge fine structure of the carbon ionization edge. The methodology used follows that of Urbonaite and JeanneRose. $^{61,62}$ Deconvolution of bands in the EELS spectra in the carbon K-edge region in Figure 3 indicated strongly that the SAPO-37-templated AC had a higher fraction of $\mathrm{sp}^{2} / \mathrm{sp}^{3}$ carbon than the zeolite-Y-templated analogues as indicated below.

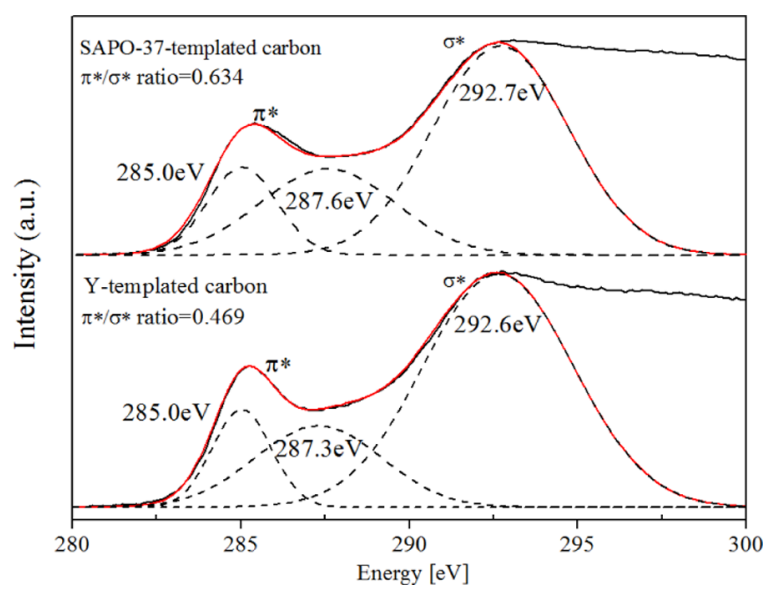

Figure 3. Deconvolution of bands in the EELS spectra in the carbon Kedge region the silicoaluminophosphate (SAPO)-37- and zeolite-Ytemplated carbons.

Figure 3 presents the EELS spectra of SAPO-37- and zeoliteY-templated ACs. The fraction of $\mathrm{sp}^{2} / \mathrm{sp}^{3}$ carbon of these two curves has been shown to be related to the hybridization of the carbon atoms, and a series of Gaussian peaks were fitted to the deconvolved, background-subtracted $\mathrm{C}-\mathrm{K}$ edge under the $\sigma^{*}$ and $\pi^{*}$ edges based on the method reported by Papworth et al. ${ }^{63}$ Quantitative calculations show that the spectrum of the SAPO37-templated AC has a statistically higher ratio for the $\pi^{*}$ peak to the $\sigma^{*}$ peak, namely, the fraction of $\mathrm{sp}^{2} / \mathrm{sp}^{3}$ carbon, $(0.634 \pm$ $0.002)$ than that of zeolite-Y-templated AC $(0.469 \pm 0.001)$. While we do not have access to the absolute $\mathrm{sp}^{2} / \mathrm{sp}^{3}$ hybridization for this study due to the lack of a standard, we do observe an obvious increase in the $\pi^{*} / \sigma^{*}$ ratio for the SAPO37-templated and zeolite-Y-templated ACs than that for the amorphous carbon (Figure S3). We interpret this increase as a higher fraction of $\mathrm{sp}^{2} / \mathrm{sp}^{3}$ carbon of these two ACs compared to that of the amorphous carbon.

TGA was performed to study how well the CVD procedure had proceeded and the thermal stability of the ACs. Analysis of the TGA traces recorded in dry air for both the SAPO-37 and zeolite Y AC composites yields carbon fractions of 0.2 and $0.3 \mathrm{~g} /$ $\mathrm{g}$ in these composites, respectively. The higher fraction of carbon with zeolite $\mathrm{Y}$ could relate to difference in particle size or chemistry.

The carbon matrix was oxidized at a comparably high temperature of $\sim 650{ }^{\circ} \mathrm{C}$ (Figure $4 \mathrm{a}, \mathrm{b}$ ), and the high temperature was consistent with carbon deposited within the

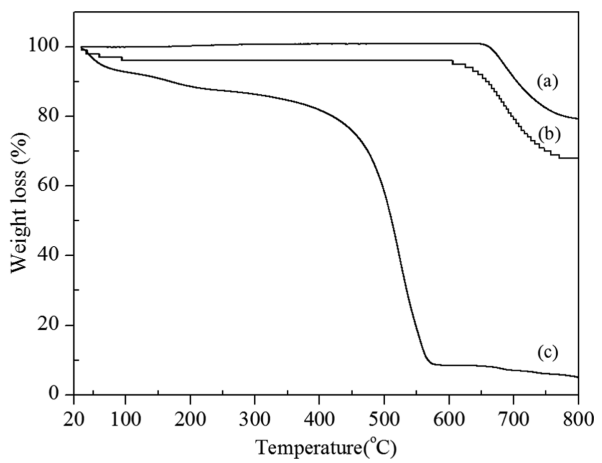

Figure 4. Thermogravimetric analysis traces of (a) silicoaluminophosphate (SAPO)-37-activated carbon (AC) composite, (b) zeolite-Y-AC composite, and (c) SAPO-37-templated AC after the dissolution of SAPO-37.

nanometer-sized cages of the templates. The TGA trace of the SAPO-37-templated AC (Figure 4c), recorded in air, indicates that oxidation reactions occur mainly at temperatures of 450$550{ }^{\circ} \mathrm{C}$; the $\mathrm{AC}$ has a relatively high thermal oxidation stability. Less than 5 wt \% of ash was left after oxidizing the SAPO-37templated AC in the TG instrument. This low ash amount was consistent with the ICP-OES elemental analysis result of the SAPO-37-templated AC (Table S2 in the Supporting Information), which shows that there was only a minor trace of the SAPO-37 matrix in the sample.

Raman and IR spectroscopies were used to study aspects of the ordering of the carbon domains and functional groups in the ACs. The SAPO-37- and zeolite-Y-templated ACs had Raman and IR spectra with typical bands for carbon materials. ${ }^{64,65}$ In the Raman spectra of both ACs (Figure 5a), two distinctive Raman bands were observed. The $\mathrm{G}$ band at around 1580-1600 $\mathrm{cm}^{-1}$ can be described as $\mathrm{C}-\mathrm{C}$ stretching, whereas the $\mathrm{D}$ band at around $1350 \mathrm{~cm}^{-1}$ represents a global vibration mode of the aromatic rings. ${ }^{65}$ The peak broadening in the Raman spectral bands indicates an atomically disordered arrangement of the carbon atoms. The IR spectra of both ACs (Figure 5b) showed band features assigned to $-\mathrm{OH}$ (around $3400 \mathrm{~cm}^{-1}$ ) and carbonyl groups (around $\left.1600 \mathrm{~cm}^{-1}\right) .{ }^{63}$ For the SAPO-37templated AC, the band-related feature at $\sim 1300 \mathrm{~cm}^{-1}$ in Figure $5 \mathrm{~b}$ is consistent with vibrational bands of phosphate or polyphosphate moieties. ${ }^{66}$

The $\mathrm{N}_{2}$ adsorption-desorption measurements were carried to measure the surface area and porosity of SAPO-37- and zeolite-Y-templated ACs. As is shown in Table 1, both the SAPO-37- and zeolite-Y-templated ACs display high specific surface areas and relatively large micropore volumes, with the zeolite-Y-templated AC displaying larger values. The shapes of the $\mathrm{N}_{2}$ isotherms (Figure 6a) of the SAPO-37- and zeolite-Ytemplated ACs differ slightly. The isotherm for the zeolite-Ytemplated AC has a clear microporosity combined with a hysteresis loop at $p / p_{0}=0.4-1.0$, typical for a measurable fraction of mesopores with a wide pore size distribution. The isotherm for the SAPO-37-templated AC is of a type I microporous material.

The adsorption and desorption of $\mathrm{CO}_{2}$ at $0{ }^{\circ} \mathrm{C}$ are typically studied for ACs to access the influence of pores smaller than those accessed by $\mathrm{N}_{2}$. As shown in Figure $6 \mathrm{~b}$, the SAPO-37templated $\mathrm{AC}$ has a higher uptake of $\mathrm{CO}_{2}$ than the zeolite-Ytemplated AC, typical for a material with a larger volume of ultramicropores. 

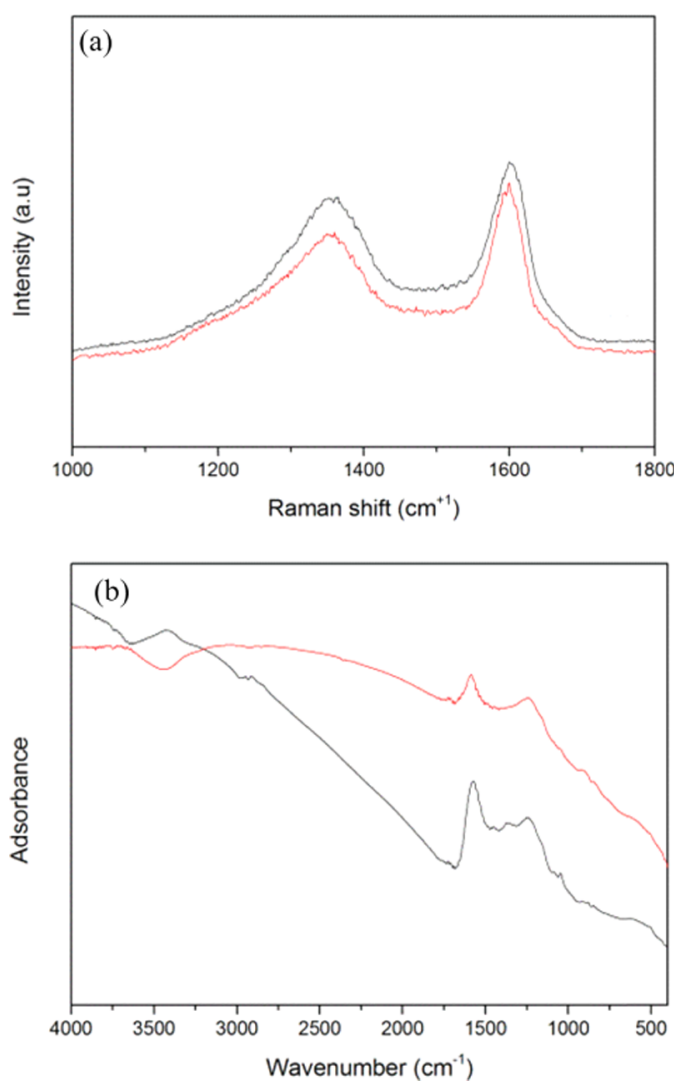

Figure 5. (a) Raman and (b) IR spectra of silicoaluminophosphate (SAPO)-37-templated (black) and zeolite-Y-templated (red) activated carbons.

Table 1. Surface Areas and Pore Volumes of Silicoaluminophosphate (SAPO)-37- and Zeolite-YTemplated Activated Carbons (ACs)

$\begin{array}{cccc}\text { sample } & S_{\text {BET }}\left(\mathrm{m}^{2} / \mathrm{g}\right) & V_{\text {micro }}\left(\mathrm{cm}^{3} / \mathrm{g}\right) & S_{\text {meso }}\left(\mathrm{m}^{2} / \mathrm{g}\right) \\ \text { Zeolite-Y-templated AC } & 2460 & 0.6 & 966 \\ \text { SAPO-37-templated AC } & 1690 & 0.4 & 705\end{array}$

The pore size distributions (PSDs) displayed in Figure 7 were calculated by density functional theory (DFT) method under the assumption of slit-shaped pores. The SAPO-37-templated AC had a near identical micropore and ultramicropore distributions for the zeolite-Y-templated AC. The PSDs derived from the $\mathrm{N}_{2}$ adsorption data at $-196{ }^{\circ} \mathrm{C}$ is shown in Figure $7 \mathrm{a}$. From these traces, it can be seen that the SAPO-37-templated $\mathrm{AC}$ has a similar pore size in the range of $1-2 \mathrm{~nm}$ as compared to that of the zeolite-Y-templated AC. The PSDs of ultrasmall micropores were derived from $\mathrm{CO}_{2}$ adsorption data recorded at $0{ }^{\circ} \mathrm{C}$. As is shown in Figure $7 \mathrm{~b}$, the SAPO-37-templated AC had a larger amount of ultramicropores in the range of $0.4-0.7 \mathrm{~nm}$ than the zeolite-Y-templated AC.

\section{CONCLUSIONS}

A microporous AC with the interior replica of the micropores of a SAPO template was synthesized for the first time. The SAPO37-templated AC had a high specific surface area, large micropore volume, and a large volume of ultrasmall micropores. With its well-defined and only micropores, the SAPO-37templated AC could be important to certain gas separation processes, where kinetic separation or even molecular sieving is
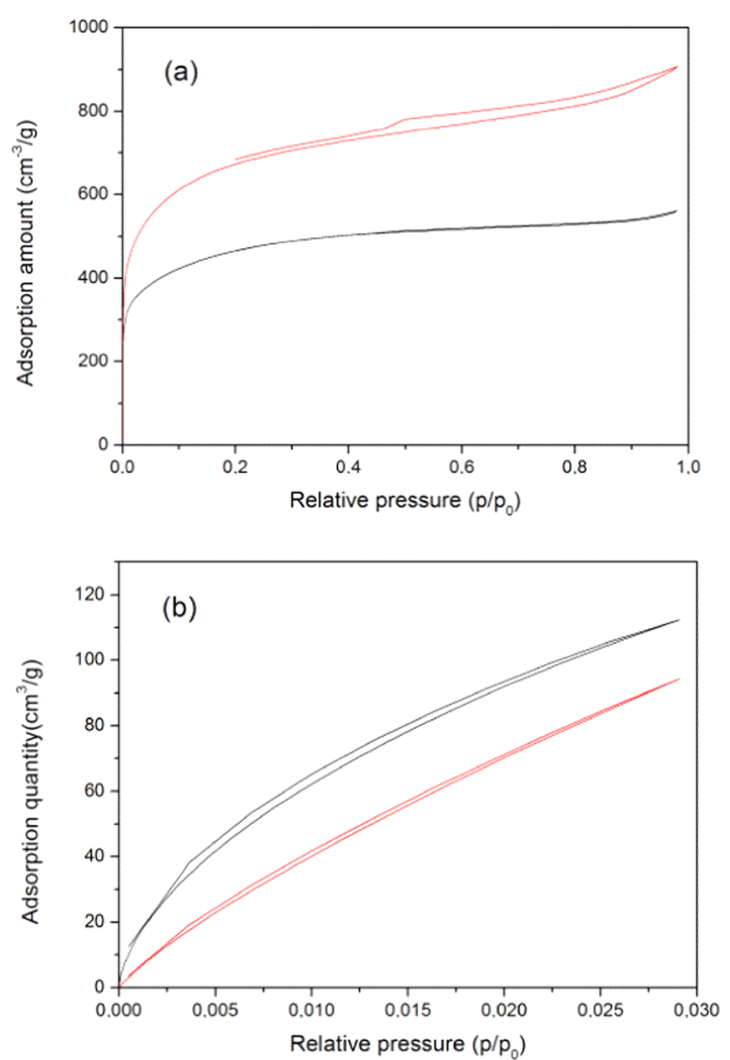

Figure 6. (a) $\mathrm{N}_{2}$ adsorption and desorption isotherms recorded at $-196{ }^{\circ} \mathrm{C}$, and (b) $\mathrm{CO}_{2}$ adsorption and desorption isotherms recorded at $0{ }^{\circ} \mathrm{C}$ on a silicoaluminophosphate (SAPO)-templated activated carbon (AC, black) and a zeolite-Y-templated AC (red).

of importance, and with a potential of higher electric conductivity than regular ACs prepared at a similar temperature, it has the potential in electrochemical applications and in supercapacitors. SAPO-37-templated AC would have similar advantages over regular ACs as the very well studied zeolite $\mathrm{Y}$ template ones, but the SAPO template could be removed without the use of $\mathrm{HF}$, which could have positive ramifications for its industrial adaptation. This first example of a SAPO-37templated microporous AC with SAPO's interior replica could open up for further studies in relation to various applications of such carbons. Open questions remain with respect to the role of phosphorous atoms during the CVD and the thermal annealing steps, which could motivate in situ studies of the related hightemperature chemistry. As microporous $\mathrm{SAPO}$ and $\mathrm{AlPO}_{4}$ framework materials have a large variety of structures, we expect further options for preparing a relatively wide array of porous templated carbons and ACs with new structures and functionalities.

\section{EXPERIMENTAL SECTION}

4.1. Materials. Tetrapropylammonium hydroxide (TPAOH, 40 wt \% in water) was from Alfa Aesar. Phosphoric acid ( 85 wt \% in water) and tetramethylammonium hydroxide (25 wt \% in water) were from Sigma Aldrich, and 2 vol \% propylene in $\mathrm{N}_{2}$ (Strandmöllen) was used as received. The reference material zeolite $\mathrm{Y}(\mathrm{H}$ form, $\mathrm{Si} / \mathrm{Al}=30)$ was purchased from Zeolyst.

4.2. Synthesis of SAPO-37. SAPO-37 was synthesized according to the reported procedure. ${ }^{67}$ A gel was prepared with a composition of 0.05 tetramethylammonium: 2 tetrapropylam- 

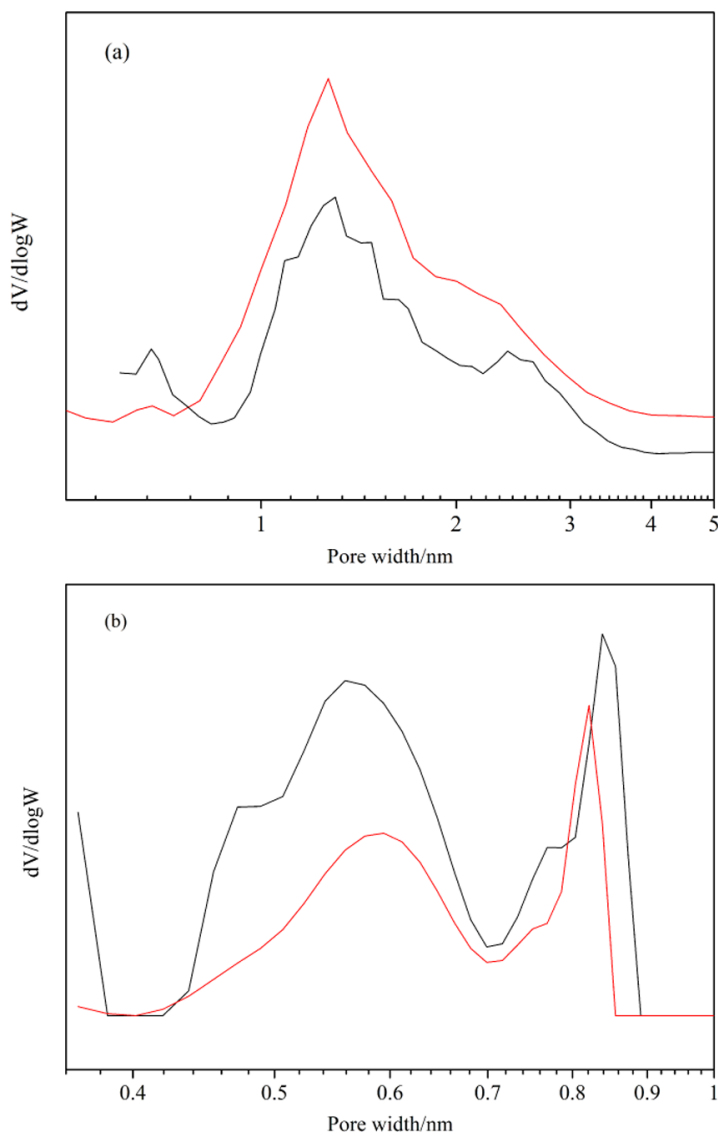

Figure 7. Pore size distributions calculated by the DFT method from (a) $\mathrm{N}_{2}$ adsorption data recorded at $-196{ }^{\circ} \mathrm{C}$ and (b) $\mathrm{CO}_{2}$ adsorption data recorded at $0{ }^{\circ} \mathrm{C}$. Data for the silicoaluminophosphate (SAPO)37-templated activated carbon (AC) are indicated with black traces and for the zeolite-Y-templated AC in red.

monium: $\mathrm{Al}_{2} \mathrm{O}_{3}: \mathrm{P}_{2} \mathrm{O}_{5}: 0.5 \mathrm{SiO}_{2}: 50 \mathrm{H}_{2} \mathrm{O}$. The gel was homogenized and transferred into Teflon-lined autoclaves and heated at a temperature of $200{ }^{\circ} \mathrm{C}$ for $16 \mathrm{~h}$. Subsequently, the autoclaves were quenched with cold water. The solids were centrifuged and washed with distilled water.

4.3. Deposition of Carbons. Carbon was deposited and pyrolyzed in the SAPO-37 and zeolite Y templates by chemical vapor deposition (CVD) with propylene using a procedure similar to an earlier reported method. Furfuryl alcohol impregnation/polymerization was not used. ${ }^{43}$ As-made SAPO37 (with the TPAOH template) was compressed into a pellet with a diameter of $2 \mathrm{~cm}$. This pellet was loaded into a vertical tube furnace with a quartz reactor, and the organic template in the as-made SAPO- 37 was pyrolyzed in a flow of $\mathrm{N}_{2}(100 \mathrm{~mL} /$ min) at a temperature of $600{ }^{\circ} \mathrm{C}$ for $12 \mathrm{~h}$. Subsequently, the temperature was raised to $800{ }^{\circ} \mathrm{C}$, after which the gas was switched to dilute propylene in $\mathrm{N}_{2}$. After propylene CVD for $4 \mathrm{~h}$, the SAPO-37-AC composite was rigidified by heat treatment in a flow of $\mathrm{N}_{2}$ at a temperature of $900{ }^{\circ} \mathrm{C}$. As a reference material, a zeolite-Y-AC composite was prepared using the same procedure.

4.4. Recovery of Carbons by Dissolution of SAPO-37 and Zeolite Y. The SAPO-37-AC composite ( $0.5 \mathrm{~g})$ was successively washed with aqueous solutions of $2 \mathrm{~mol} / \mathrm{dm}^{3} \mathrm{HCl}$ and then $2 \mathrm{~mol} / \mathrm{dm}^{3} \mathrm{NaOH}$ solutions at $60{ }^{\circ} \mathrm{C}$. The remaining solids were recovered by centrifugation and subsequently washed with distilled water. An attempt to dissolve the zeolite $\mathrm{Y}$ template with the same procedure was unsuccessful. Instead, the zeolite-Y-AC composite was dissolved using an aqueous solution of $2 \mathrm{~mol} / \mathrm{dm}^{3} \mathrm{HCl}$ and $2 \mathrm{~mol} / \mathrm{dm}^{3} \mathrm{HF}$ at $60^{\circ} \mathrm{C}$. The remaining solids were also recovered by centrifugation and subsequent washing with distilled water.

4.5. Characterization. Powder X-ray diffraction (PXRD) data was collected on a PANalytical X'Pert Pro diffractometer using $\mathrm{Cu} \mathrm{K} \alpha$ radiation $(\lambda=1.5418 \AA ̊ \AA)$. Morphologies were examined by scanning electron microscopy (SEM) using a JEOL JSM-7000 microscope with an accelerating voltage of $15 \mathrm{kV}$. The transmission electron microscopy (TEM) and highresolution TEM (HRTEM) experiments were performed on a JEOL JEM-2100F field-emission electron microscope, operating at $200 \mathrm{kV}$. Electron energy-loss spectroscopy (EELS) was performed using a postcolumn Gatan Image Filter (GIF Tridium), whereas the TEM was operated in scanning TEM (STEM) mode. Further experimental details are provided in the Supporting Information. The elemental compositions were determined with TEM energy-dispersive X-ray spectroscopy (EDS). For the TEM, EELS, and EDS experiments, powders of the samples were ultrasonically dispersed in ethanol at room temperature for $2 \mathrm{~min}$ and then transferred onto a grid by dipping (continuous carbon-coated copper grids were used for TEM images, and holy carbon-coated copper grids were used for EELS and EDS). Thermogravimetric analysis (TGA) was conducted in a flow of dry air with a TA Instruments Discovery TG. The samples were heated from room temperature to $800^{\circ} \mathrm{C}$ at a heating rate of $10{ }^{\circ} \mathrm{C} / \mathrm{min}$. Basic elemental content of $\mathrm{C}, \mathrm{H}$, and $\mathrm{N}$ was measured by combustion analysis, and the possible elements from the templates were measured by ICP-OES following acid digestion (nitric and HF acid). A Varian 670 Fourier transform infrared (FT-IR) spectrometer was used to record IR spectra in a transmission mode using a $\mathrm{KBr}$-pellet method. The Raman spectra were recorded with a LabRAM HR 800 instrument using a $532 \mathrm{~nm}$ laser. The porosities were analyzed with a Micromeritics ASAP 2020 instrument using $\mathrm{N}_{2}$ adsorption data recorded at a temperature of $-196^{\circ} \mathrm{C}$, and $\mathrm{CO}_{2}$ adsorption data were recorded at $0^{\circ} \mathrm{C}$. Samples were degassed at $200{ }^{\circ} \mathrm{C}$ for $6 \mathrm{~h}$ before the adsorption experiments. The surface areas were calculated by the Brunauer-Emmett-Teller (BET) method using the $\mathrm{N}_{2}$-adsorption data. The relative pressures used for the BET method were selected ensuring a positive intersection with the $y$-axis and a Rouquerol transformation that increased with the relative pressure. ${ }^{68}$ The pore size distributions were calculated by a density functional theory (DFT) assuming a slit-shaped pore geometry.

\section{ASSOCIATED CONTENT}

\section{S Supporting Information}

The Supporting Information is available free of charge on the ACS Publications website at DOI: 10.1021/acsomega.9b00135.

Scanning electron microscopy and HRTEM images of SAPO-37-templated AC; TEM-EDS spectra of the SAPO-37- and zeolite-Y-templated ACs; EELS spectra in the carbon K-edge region from the SAPO-37-AC, zeolite-Y-templated $\mathrm{AC}$, and the amorphous carbon; elemental content in the SAPO-37- and zeolite-Ytemplated AC; and their analysis (PDF)

\section{AUTHOR INFORMATION}

\section{Corresponding Author}

*E-mail: niklas.hedin@mmk.su.se. 


\section{ORCID $\odot$}

Niklas Hedin: 0000-0002-7284-2974

\section{Author Contributions}

${ }^{\dagger}$ Y.L. and X.W. contributed equally.

\section{Author Contributions}

The manuscript was written through contributions of all authors. All authors have given approval to the final version of the manuscript.

\section{Funding}

This project was supported by the Swedish Energy Agency and the EU-MSCA-ETN-GreenCarbon project 721991.

\section{Notes}

The authors declare no competing financial interest.

\section{REFERENCES}

(1) Lalvani, S. B.; Wiltowski, T.; Hübner, A.; Weston, A.; Mandich, N. Removal of Hexavalent Chromium and Metal Cations by a Selective and Novel Carbon Adsorbent. Carbon 1998, 36, 1219-1226.

(2) Hedin, N.; Andersson, L.; Bergström, L.; Yan, J. Adsorbents for the Post-Combustion Capture of $\mathrm{CO}_{2}$ Using Rapid Temperature Swing or Vacuum Swing Adsorption. Appl. Energy 2013, 104, 418-433.

(3) Portet, C.; Taberna, P. L.; Simon, P.; Flahaut, E.; Laberty-Robert, C. High Power Density Electrodes for Carbon Supercapacitor Applications. Electrochim. Acta 2005, 50, 4174-4181.

(4) Ma, Z.; Kyotani, T.; Liu, Z.; Terasaki, O.; Tomita, A. Very High Surface Area Microporous Carbon with a Three-Dimensional NanoArray Structure: Synthesis and Its Molecular Structure. Chem. Mater. 2001, 13, 4413-4415.

(5) Itoi, H.; Nishihara, H.; Kogure, T.; Kyotani, T. ThreeDimensionally Arrayed and Mutually Connected 1.2-Nm Nanopores for High-Performance Electric Double Layer Capacitor. J. Am. Chem. Soc. 2011, 133, 1165-1167.

(6) Gu, W.; Yushin, G. Review of Nanostructured Carbon Materials for Electrochemical Capacitor Applications: Advantages and Limitations of Activated Carbon, Carbide-Derived Carbon, ZeoliteTemplated Carbon, Carbon Aerogels, Carbon Nanotubes, Onion-like Carbon, and Graphene. Wiley Interdiscip. Rev.: Energy Environ. 2014, 3, 424-473.

(7) Ania, C. O.; Khomenko, V.; Raymundo-Piñero, E.; Parra, J. B.; Béguin, F. The Large Electrochemical Capacitance of Microporous Doped Carbon Obtained by Using a Zeolite Template. Adv. Funct. Mater. 2007, 17, 1828-1836.

(8) Kim, K.; Choi, M.; Ryoo, R. Ethanol-Based Synthesis of Hierarchically Porous Carbon Using Nanocrystalline Beta Zeolite Template for High-Rate Electrical Double Layer Capacitor. Carbon 2013, 60, 175-185.

(9) Hou, P.-X.; Yamazaki, T.; Orikasa, H.; Kyotani, T. An Easy Method for the Synthesis of Ordered Microporous Carbons by the Template Technique. Carbon 2005, 43, 2624-2627.

(10) Pacuła, A.; Mokaya, R. Synthesis and High Hydrogen Storage Capacity of Zeolite-Like Carbons Nanocast Using As-Synthesized Zeolite Templates. J. Phys. Chem. C 2008, 112, 2764-2769.

(11) Ma, Z.; Kyotani, T.; Tomita, A. Synthesis Methods for Preparing Microporous Carbons with a Structural Regularity of Zeolite Y. Carbon 2002, 40, 2367-2374.

(12) Baerlocher, Ch.; McCusker, L. B. Database of Zeolite Structures: http://www.iza-structure.org/databases/(accessed Dec.4 9, 2018)

(13) Atlas of Zeolite Framework Types, 5th rev. ed.; Baerlocher, C., Meier, W. M., Olson, D., Meier, W. M., Eds.; Elsevier: New York, 2001.

(14) Parmentier, J.; Gaslain, F. O. M.; Ersen, O.; Centeno, T. A.; Solovyov, L. A. Structure and Sorption Properties of a ZeoliteTemplated Carbon with the EMT Structure Type. Langmuir 2014, 30, 297-307.

(15) Kim, K.; Lee, T.; Kwon, Y.; Seo, Y.; Song, J.; Park, J. K.; Lee, H.; Park, J. Y.; Ihee, H.; Cho, S. J.; et al. Lanthanum-Catalysed Synthesis of Microporous 3D Graphene-like Carbons in a Zeolite Template. Nature 2016, 535, 131-135.
(16) Kyotani, T.; Ma, Z.; Tomita, A. Template Synthesis of Novel Porous Carbons Using Various Types of Zeolites. Carbon 2003, 41, 1451-1459.

(17) Johnson, S. A.; Brigham, E. S.; Ollivier, P. J.; Mallouk, T. E. Effect of Micropore Topology on the Structure and Properties of Zeolite Polymer Replicas. Chem. Mater. 1997, 9, 2448-2458.

(18) Gaslain, F. O. M.; Parmentier, J.; Valtchev, V. P.; Patarin, J. First Zeolite Carbon Replica with a Well Resolved X-Ray Diffraction Pattern. Chem. Commun. 2006, 0, 991-993.

(19) Inagaki, M.; Kang, F.; Toyoda, M.; Konno, H. Template Carbonization: Morphology and Pore Control. Advanced Materials Science and Engineering of Carbon; Inagaki, M., Kang, F., Toyoda, M., Konno, H., Eds.; Butterworth-Heinemann: Boston, 2014; Chapter 7, pp $133-163$.

(20) Hartman, R. L.; Fogler, H. S. Understanding the Dissolution of Zeolites. Langmuir 2007, 23, 5477-5484.

(21) Li, Y.; Zheng, H.; Yun, Y.; Bernin, D.; Edén, M.; Zou, X.; Gao, F. De-Agglomeration of IZM-2 Zeolite Crystals by Post-Synthetic Treatment. Microporous Mesoporous Mater. 2016, 225, 185-191.

(22) Van Tendeloo, L.; Gobechiya, E.; Breynaert, E.; Martens, J. A.; Kirschhock, C. E. A. Alkaline Cations Directing the Transformation of FAU Zeolites into Five Different Framework Types. Chem. Commun. 2013, 49, 11737-11739.

(23) Wilson, S. T.; Lok, B. M.; Messina, C. A.; Cannan, T. R.; Flanigen, E. M. Aluminophosphate Molecular Sieves: A New Class of Microporous Crystalline Inorganic Solids. J. Am. Chem. Soc. 1982, 104, $1146-1147$

(24) Davis, M. E. Ordered Porous Materials for Emerging Applications. Nature 2002, 417, 813-821.

(25) Winiecki, A. M.; Suib, S. L. Chemical Stability of Aluminophosphate Molecular Sieves during $\mathrm{HCl}$ Treatment. Langmuir 1989, 5, 333-338.

(26) Liu, G.; Liu, Y.; Zhang, X.; Yuan, X.; Zhang, M.; Zhang, W.; Jia, M. Characterization and Catalytic Performance of Porous Carbon Prepared Using in Situ-Formed Aluminophosphate Framework as Template. J. Colloid Interface Sci. 2010, 342, 467-473.

(27) Tang, Z. K.; Wang, N.; Zhang, X. X.; Wang, J. N.; Chan, C. T.; Sheng, P. Novel Properties of $0.4 \mathrm{Nm}$ Single-Walled Carbon Nanotubes Templated in the Channels of $\mathrm{AlPO}_{4}-5$ Single Crystals. New J. Phys. 2003, 5, 146.

(28) Lok, B. M.; Messina, C. A.; Patton, R. L.; Gajek, R. T.; Cannan, T. R.; Flanigen, E. M. Silicoaluminophosphate Molecular Sieves: Another New Class of Microporous Crystalline Inorganic Solids. J. Am. Chem. Soc. 1984, 106, 6092-6093.

(29) Chen, L.; Singh, R. K.; Webley, P. Synthesis, Characterization and Hydrogen Storage Properties of Microporous Carbons Templated by Cation Exchanged Forms of Zeolite Y with Propylene and Butylene as Carbon Precursors. Microporous Mesoporous Mater. 2007, 102, 159170

(30) Matsuoka, K.; Yamagishi, Y.; Yamazaki, T.; Setoyama, N.; Tomita, A.; Kyotani, T. Extremely High Microporosity and Sharp Pore Size Distribution of a Large Surface Area Carbon Prepared in the Nanochannels of Zeolite Y. Carbon 2005, 43, 876-879.

(31) Yang, Z.; Xia, Y.; Sun, X.; Mokaya, R. Preparation and Hydrogen Storage Properties of Zeolite-Templated Carbon Materials Nanocast via Chemical Vapor Deposition: Effect of the Zeolite Template and Nitrogen Doping. J. Phys. Chem. B 2006, 110, 18424-18431.

(32) Alam, N.; Mokaya, R. Evolution of Optimal Porosity for Improved Hydrogen Storage in Templated Zeolite-like Carbons. Energy Environ. Sci. 2010, 3, 1773-1781.

(33) Ma, Z.; Kyotani, T.; Tomita, A. Preparation of a High Surface Area Microporous Carbon Having the Structural Regularity of $\mathrm{Y}$ Zeolite. Chem. Commun. 2000, 0, 2365-2366.

(34) Kyotani, T.; Nagai, T.; Inoue, S.; Tomita, A. Formation of New Type of Porous Carbon by Carbonization in Zeolite Nanochannels. Chem. Mater. 1997, 9, 609-615.

(35) Lok, B. M.; Messina, C. A.; Patton, R. L.; Gajek, R. T.; Cannan, T. R.; Flanigen, E. M. Crystalline Silicoaluminophosphates. U.S. Patent $4,440,8711984$ 
(36) Baerlocher, Ch.; McCusker, L. B. Database of Zeolite Structures: http://www.iza-structure.org/databases/ (accessed Mar 9, 2017).

(37) Dzwigaj, S.; Briend, M.; Shikholeslami, A.; Peltre, M. J.; Barthomeuf, D. The Acidic Properties of SAPO-37 Compared to Faujasites and SAPO-5. Zeolites 1990, 10, 157-162.

(38) Briend, M.; Shikholeslami, A.; Peltre, M.-J.; Delafosse, D.; Barthomeuf, D. Thermal and Hydrothermal Stability of SAPO-5 and SAPO-37 Molecular Sieves. J. Chem. Soc., Dalton Trans. 1989, 0, 13611362.

(39) Mu, Y.; Shi, H.; Wang, Y.; Ding, H.; Li, J. CNDs@zeolite: New Room-Temperature Phosphorescent Materials Derived by Pyrolysis of Organo-Templated Zeolites. J. Mater. Chem. C 2017, 5, 10894-10899.

(40) Mu, Y.; Wang, N.; Sun, Z.; Wang, J.; Li, J.; Yu, J. Carbogenic Nanodots Derived from Organo-Templated Zeolites with Modulated Full-Color Luminescence. Chem. Sci. 2016, 7, 3564-3568.

(41) Wu, X.; Hong, X.; Nan, J.; Luo, Z.; Zhang, Q.; Li, L.; Chen, H.; Hui, K. S. Electrochemical Double-Layer Capacitor Performance of Novel Carbons Derived from SAPO Zeolite Templates. Microporous Mesoporous Mater. 2012, 160, 25-31.

(42) Ma, Z.; Kyotani, T.; Tomita, A. Synthesis Methods for Preparing Microporous Carbons with a Structural Regularity of Zeolite Y. Carbon 2002, 40, 2367-2374.

(43) Ma, Z.; Kyotani, T.; Tomita, A. Preparation of a High Surface Area Microporous Carbon Having the Structural Regularity of $\mathrm{Y}$ Zeolite. Chem. Commun. 2000, 0, 2365-2366.

(44) Nueangnoraj, K.; Nishihara, H.; Imai, K.; Itoi, H.; Ishii, T.; Kiguchi, M.; Sato, Y.; Terauchi, M.; Kyotani, T. Formation of Crosslinked-Fullerene-like Framework as Negative Replica of Zeolite Y. Carbon 2013, 62, 455-464.

(45) Zhao, C.; Yang, Y.; Chen, W.; Wang, H.; Zhao, D.; Webley, P. A. Hydrothermal Synthesis of Novel AlPO4-5 Brooms and Nano-Fibers and Their Templated Carbon Structures. CrystEngComm 2009, 11, 739.

(46) Harris, P. J. F.; Burian, A.; Duber, S. High-Resolution Electron Microscopy of a Microporous Carbon. Philos. Mag. Lett. 2000, 80, 381386.

(47) Harris, P. J. F.; Tsang, S. C.; Claridge, J. B.; Green, M. L. H. HighResolution Electron Microscopy Studies of a Microporous Carbon Produced by Arc-Evaporation. J. Chem. Soc., Faraday Trans. 1994, 90, 2799-2802.

(48) Harris, P. J. F.; Tsang, S. C. High-Resolution Electron Microscopy Studies of Non-Graphitizing Carbons. Philos. Mag. A 1997, 76, 667-677.

(49) Harris, J. F.; Vis, R. D. High-Resolution Transmission Electron Microscopy of Carbon and Nanocrystals in the Allende Meteorite. Proc. R. Soc. Lond. A 2003, 459, 2069-2076.

(50) Terzyk, A. P.; Furmaniak, S.; Harris, P. J. F.; Gauden, P. A.; Włoch, J.; Kowalczyk, P.; Rychlicki, G. How Realistic Is the Pore Size Distribution Calculated from Adsorption Isotherms If Activated Carbon Is Composed of Fullerene-like Fragments? Phys. Chem. Chem. Phys. 2007, 9, 5919-5927.

(51) Ōya, A.; Mochizuki, M.; Ōtani, S.; Tomizuka, I. An Electron Microscopic Study on the Turbostratic Carbon Formed in Phenolic Resin Carbon by Catalytic Action of Finely Dispersed Nickel. Carbon 1979, 17, 71-76.

(52) Wollbrink, A.; Volgmann, K.; Koch, J.; Kanthasamy, K.; Tegenkamp, C.; Li, Y.; Richter, H.; Kämnitz, S.; Steinbach, F.; Feldhoff, A.; et al. Amorphous, Turbostratic and Crystalline Carbon Membranes with Hydrogen Selectivity. Carbon 2016, 106, 93-105.

(53) Scheffler, M.; Greil, P.; Berger, A.; Pippel, E.; Woltersdorf, J. Nickel-Catalyzed in Situ Formation of Carbon Nanotubes and Turbostratic Carbon in Polymer-Derived Ceramics. Mater. Chem. Phys. 2004, 84, 131-139.

(54) Li, Z. Q.; Lu, C. J.; Xia, Z. P.; Zhou, Y.; Luo, Z. X-Ray Diffraction Patterns of Graphite and Turbostratic Carbon. Carbon 2007, 45, 16861695.

(55) Radovic, L., Ed. Chemistry \& Physics of Carbon; CRC Press: Boca Raton, 2003.
(56) Setton, R., Bernier, P., Lefrant, S., Eds. Carbon Molecules and Materials; CRC Press: London, 2002.

(57) Titantah, J.; Lamoen, D. Technique for the Sp2/sp3 Characterization of Carbon Materials: $\mathrm{Ab}$ Initio Calculation of near-Edge Structure in Electron-Energy-Loss Spectra. Phys. Rev. B 2004, 70, No. 075115.

(58) Brydson, R. Electron Energy Loss Spectroscopy; BIOS Sci Publ.: Oxford, 2001.

(59) Berger, S. D.; McKenzie, D. R.; Martin, P. J. EELS Analysis of Vacuum Arc-Deposited Diamond-like Films. Philos. Mag. Lett. 1988, 57, 285-290.

(60) Mykhaylyk, O. O.; Solonin, Y. M.; Batchelder, D. N.; Brydson, R. Transformation of Nanodiamond into Carbon Onions: A Comparative Study by High-Resolution Transmission Electron Microscopy, Electron Energy-Loss Spectroscopy, $\mathrm{x}$-Ray Diffraction, Small-Angle $\mathrm{X}$-Ray Scattering, and Ultraviolet Raman Spectroscopy. J. Appl. Phys. 2005, 97, No. 074302.

(61) Jeanne-Rose, V.; Golabkan, V.; Mansot, J. L.; Largitte, L.; Césaire, T.; Ouensanga, A. An EELS-Based Study of the Effects of Pyrolysis on Natural Carbonaceous Materials Used for Activated Charcoal Preparation. J. Microsc. 2003, 210, 53-59.

(62) Urbonaite, S.; Wachtmeister, S.; Mirguet, C.; Coronel, E.; Zou, W. Y.; Csillag, S.; Svensson, G. EELS Studies of Carbide Derived Carbons. Carbon 2007, 45, 2047-2053.

(63) Papworth, A. J.; Kiely, C. J.; Burden, A. P.; Silva, S. R. P.; Amaratunga, G. A. J. Electron-Energy-Loss Spectroscopy Characterization of the sp2 Bonding Fraction within Carbon Thin Films. Phys. Rev. B 2000, 62, 12628-12631.

(64) Ferrari, A. C. Raman Spectroscopy of Graphene and Graphite: Disorder, Electron-Phonon Coupling, Doping and Nonadiabatic Effects. Solid State Ionics 2007, 143, 47-57.

(65) Nishihara, H.; Yang, Q.-H.; Hou, P.-X.; Unno, M.; Yamauchi, S.; Saito, R.; Paredes, J. I.; Martínez-Alonso, A.; Tascón, J. M. D.; Sato, Y. A Possible Buckybowl-like Structure of Zeolite Templated Carbon. Carbon 2009, 47, 1220-1230.

(66) Puziy, A. M.; Poddubnaya, O. I.; Martínez-Alonso, A.; SuárezGarcía, F.; Tascón, J. M. Synthetic Carbons Activated with Phosphoric Acid: I. Surface Chemistry and Ion Binding Properties. Carbon 2002, $40,1493-1505$.

(67) Sierra de Saldarriaga, L.; Saldarriaga, C.; Davis, M. E. Investigations into the Nature of a Silicoaluminophosphate with the Faujasite Structure. J. Am. Chem. Soc. 1987, 109, 2686-2691.

(68) Determination of the specific surface area of solids by gas adsorption-BET method. International Standard ISO 9277:2010(E), second edition 2010.09.01. 